\title{
AVALIAÇÃO ISOCINÉTICA EM ATLETAS DA SELEÇÃO BRASILEIRA DE FUTEBOL DE 5
}

\section{DE FÚTBOL DE 5}

Luis Felipe Castelli Correia de Campos ${ }^{1,3}$ (Educador Físico)

João Paulo Borin ${ }^{2,3}$ (Educador Físico)

Luiz Gustavo Teixeira Fabricio dos

Santos ${ }^{1}$ (Educador Físico)

Thiago Mattos Frota de Souza' (Educador Físico)

Vivian Maria dos Santos Paranhos ${ }^{1,3}$ (Nutricionista)

Ricardo Antonio Tanhoffer ${ }^{5}$

(Educador Físico)

Cristian Rocha Luarte ${ }^{4}$

(Educador Físico)

José Irineu Gorla' (Educador Físico)

1. Universidade Estadual de Campinas, Faculdade de Educação Física, Departamento de Atividade Física Adaptada. Campinas, SP, Brasil. 2. Universidade Estadual de Campinas, Faculdade de Educação Física, Departamento de Ciências do Esporte. Campinas, SP, Brasil.

3. Confederação Brasileira de Desportos para Deficientes Visuais CBDV, Rio de Janeiro, RJ, Brasil. 4. Universidad San Sebastian, Facultad de Ciencias de la Actividad Física, Concepcion, Bío-Bío, Chile. 5. Universidade Federal do Paraná, Departamento de Fisiologia, Laboratorio de Metabolismo Celular, Curitiba, PR, Brasil.

\section{Correspondência:}

Rua Gabriel de Bourbon y Bourbon, 119. São Luiz, Itu, SP, Brasil. 13300-040. pfluisfelipe@gmail.com

\section{RESUMO}

Introdução: A força muscular (FM) dos membros inferiores é um dos principais componentes exigidos para as ações específicas durante a prática do futebol de 5 e, quando apresentam níveis insuficientes, desequilíbrios bilaterais elevados ou acentuada diferença na razão agonista/antagonista (RAA) são fatores que contribuem para o desenvolvimento de lesões musculoesqueléticas. Objetivo: A proposta deste estudo foi avaliar os níveis de torque máximo, a diferença bilateral na produção de força e a razão convencional das musculaturas flexoras e extensoras do joelho em diferentes velocidades de execução. Métodos: Participaram do estudo 11 atletas deficientes visuais. Os atletas foram submetidos à avaliação antropométrica para determinação da composição corporal e submetidos à avaliação com dinamômetro isocinético para a mensuração dos níveis de desequilíbrio muscular e razão convencional. Resultados: Nos movimentos concêntricos da musculatura flexora foram observadas diferenças significativas no pico de torque (PT) entre os membros dominante (MD) e não dominante (MND) na velocidade de $60^{\circ} . \mathrm{s}^{-1}$ e $180^{\circ} . \mathrm{s}^{-1}$, no pico de torque normalizado (PTN) a $60^{\circ} . \mathrm{s}^{-1} \mathrm{e}$ na velocidade de $180^{\circ} . \mathrm{s}^{-1}$ para os músculos extensores. Na RAA, observou-se diferença significativa entre MD e MND, e níveis aceitáveis de RAA em ambas as pernas, de acordo com o proposto para o futebol convencional. Conclusão: Espera-se que os resultados do presente estudo possam contribuir para os processos de prevenção, treinamento e reabilitação de atleta de futebol de 5, como também, servirem como parâmetros para futuros estudos.

Palavras-chave: força muscular, equilibrio postural, pessoas com deficiência, dinamômetro de força muscular.

\section{ABSTRACT}

Introduction: Muscle strength (MS) of the lower limbs is one of the main components required for specific actions during practice of 5-a-side football and when the levels are insufficient, elevated bilateral imbalances or marked difference in agonist/antagonist ratio (AAR) are factors that contribute to the development of musculoskeletal injuries. Objective: The purpose of this study was to assess levels of peak torque, bilateral difference in force production and conventional ratio of flexor and extensor musculature of the knee in different speeds. Methods: The study included 11 visually impaired athletes. The athletes underwent anthropometric measurements to determine body composition and underwent evaluation at the isokinetic dynamometer to measure the levels of muscle imbalance and conventional ratio. Results: In concentric movements of the flexor muscles, significant differences were observed in peak torque (PT) between the dominant limb (DL) and non-dominant limb (NDL) at $60^{\circ} . \mathrm{S}^{-1}$ and $180^{\circ} . \mathrm{S}^{-1}$, the peak torque standardized (PTS) at $60^{\circ} . \mathrm{s}^{-1}$ and $180^{\circ} \mathrm{S}^{-1}$ to the extensor muscles. In AAR, there was significant difference between $D L$ and NDL, and acceptable levels of AAR in both legs according to the proposed for conventional football. Conclusion: It is expected that the results of this study may contribute to the processes of prevention, training and rehabilitation in athletes of 5-a-side football, also, serve as parameters for future studies.

Keywords: muscule strength, postural balance, disabled persons, muscle strength dynamometer.

\section{RESUMEN}

Introducción: La fuerza muscular (FM) de los miembros inferiores es uno de los principales componentes necesarios para acciones específicas durante la práctica de fútbol de 5 y, cuando presentan niveles insuficientes, los desequilibrios bilaterales elevados o acentuada diferencia en la razón agonista/antagonista (RAA), son factores que contribuyen para el desarrollo de lesiones musculoesqueléticas. Objetivo: En este sentido, la propuesta del estudio fue evaluar los niveles de torque máximo, diferencia bilateral en la producción de fuerza y razón convencional de las musculaturas flexoras y extensoras de la rodilla en diferentes velocidades de ejecución. Métodos: Participaron en el estudio 11 atletas con discapacidad visual. Los atletas fueron sometidos a evaluación antropométrica para determinación de la composición corporal y sometidos a evaluación en el dinamómetro isocinético para medir los niveles de desequilibrio muscular y la razón convencional. Resultados: En los movimientos concéntricos de la musculatura flexora, se observaron diferencias significativas en el pico de torque (PT) entre los miembros dominantes (MD) y no dominante (MND) en la velocidad de $60^{\circ} . \mathrm{s}^{-1}$ y $180^{\circ} \mathrm{s}^{-1}$, en el pico 
de torque normalizado (PTN) a $60^{\circ}$.s-1 y $180^{\circ}$.s-1 para los músculos extensores. En la RAA se observó diferencia significativa entre MD y MND, y niveles aceptables de RAA en ambas piernas de acuerdo con lo propuesto para el fútbol convencional. Conclusión: Se espera que los resultados del presente estudio puedan contribuir para los procesos de prevención, entrenamiento y rehabilitación de los atletas de fútbol de 5, así como también que sirvan como parámetros para futuros estudios.

Palabras clave: fuerza muscular, equilibrio postural, personas con discapacidad, dinamómetro de fuerza muscular.

\section{INTRODUÇÃO}

Devido ao comprometimento da propriocepção e elevadas cargas de treinamento, a incidência de lesões na prática esportiva é comumente observada em atletas com deficiência visual ${ }^{1}$. Dentre estas, as lesões em membros inferiores, principalmente na região do joelho e coxa, são predominantes. Por exemplo, quase $60 \%$ das lesões registradas em esportes paralímpicos, para deficientes visuais, ocorrem em membros inferiores, das quais, praticamente 50\% estão relacionadas as estruturas mencionadas. No Futebol de 5, cuja elegibilidade é para atletas cegos, a incidência de lesões nos membros inferiores é ainda maior, representando aproximadamente $80 \%$ do total de lesões ${ }^{1,2}$.

A utilização de metodologias que permitam avaliar o desequilíbrio muscular, com objetivo de prevenir lesões, torna-se assim crucial para possibilitar adequações das sessões de treinamento. Dentre estas, a avaliação isocinética é considerada padrão ouro na análise dos parâmetros neuromusculares ${ }^{3}$, e tem sido utilizada para determinar os níveis de torque produzido por atletas em ações excêntricas e concêntricas de musculaturas como quadríceps e isquiotibiais ${ }^{4,5}$, em processos de treinamento com ênfase na reabilitação pós-lesão 4 e na análise comparativa de força muscular bilateral e entre a musculatura agonista e antagonista ${ }^{6-8}$.

Porém, a investigação desses parâmetros neuromusculares, para diferentes objetivos supracitados, em atletas com deficiência visual, é escassa apesar de sua alta propensão à incidência de lesões musculares $^{1}$. Na literatura identificou-se apenas o estudo que mensurou através de avaliações de campo, a potência de membro inferior em atletas praticantes de futebol de5, através do teste de salto horizontal no momento de pré-temporada desses atletas ${ }^{9}$. O Brasil, no âmbito internacional é atualmente o campeão dos Jogos Parapanamericanos, Mundial e dos Jogos Paralímpicos. Inclusive, em 2013 recebeu o prêmio Paralympic Sport Awards, na categoria de melhor esporte coletivo em nível mundial, fato que contribui para a qualificação da amostra do estudo.

Sendo assim, no presente estudo, avaliamos o desempenho isocinético de atletas da seleção brasileira de futebol de 5, através da análise de déficits bilaterais da musculatura extensora e flexora dos joelhos, em diferentes velocidades de execução e, mensuramos a relação convencional entre a musculatura agonistas e antagonista dos membros inferiores. Espera-se que os resultados do presente estudo possam contribuir para os processos de prevenção, treinamento e reabilitação de atleta de futebol de 5, bem como, servirem como parâmetros para a relação de diferença bilateral entre os membros dominante e não dominante e a diferença de torque entre a musculatura agonista e antagonista em atletas de futebol de 5

\section{MATERIAL E MÉTODO}

Participaram do estudo 11 atletas com classificação funcional B1, que integram a seleção brasileira de futebol de 5 . Os goleiros foram excluídos da amostra pelo número reduzido de ações realizadas durante as partidas devido ao espaço limitado para atuação e, por não apresentarem deficiência visual.
No momento da coleta do estudo, os atletas encontravam-se no período preparatório geral ${ }^{10}$. Todos foram informados dos procedimentos do estudo e assinaram o termo de consentimento para a participação do estudo, de acordo com as resoluções do Comitê de Ética em Pesquisa da Faculdade de Ciências Médicas da Universidade Estadual de Campinas, SP, Brasil.

\section{Protocolo}

Antes de iniciar os procedimentos de avaliação, os atletas ficaram concentrados em ambiente restrito, no qual foram orientados e controlados para que não houvesse a realização de nenhuma atividade física moderada ou de alta intensidade nas 48h que antecederam às avaliações, evitando assim, a interferência das variáveis que pudessem comprometer os resultados dos testes.

Inicialmente, os atletas realizaram a avaliação antropométrica. A estatura (EST) foi mensurada em posição ortostática através do estadiômetro de parede da marca WCS ${ }^{\circledR}$ com precisão em 0,1 cm. A massa corporal (MC) foi mensurada por uma balança da marca Filizola ${ }^{\circledR}$, com precisão em 0,1 Kg e a mensuração das pregas cutâneas tricipital, bicipital, subescapular, peitoral, axilar, suprailíaca, abdominal, coxa e panturrilha com a utilização do adipômetro Lange ${ }^{\circledR}$ (Cambrigde, USA). A partir das medidas de EST e MC, calculamos os valores de índice de massa corporal (IMC). Os valores de pregas cutâneas foram utilizados na estimativa da densidade corporal (DC), através da equação de sete pregas cutâneas proposta por Jackson e Pollock ${ }^{11}$ e, por fim, com os valores de DC, calculou-se o percentual de gordura corporal (GC) através da equação de Siri'12.

Na sequência, os atletas foram submetidos ao protocolo no dinamômetro isocinético (Biodex System 4, Biodex Medical Systems ${ }^{\circledR}$, USA) para a mensuração dos parâmetros neuromusculares e déficits bilaterais da musculatura extensora e flexora dos joelhos, em diferentes velocidades de execução. Os atletas foram posicionados sentados na cadeira do dinamômetro de acordo com as especificações do fabricante, sendo fixados por meio de faixas no tronco, cintura e coxa. 0 eixo de rotação do dinamômetro foi alinhado visualmente ao eixo de rotação da articulação do joelho. O torque dos músculos da coxa foi assumido como sendo igual ao torque do dinamômetro corrigido pela gravidade do torque da perna.

Antes de iniciar o protocolo proposto, os indivíduos realizaram um aquecimento geral no ciclo ergômetro durante cinco minutos a $25 \mathrm{~km} / \mathrm{h}$ e, depois um aquecimento específico de cinco repetições concêntricas para extensão e flexão do joelho a $60^{\circ} . \mathrm{s}^{-1}$ no dinamômetro isocinético. Após o aquecimento, os indivíduos realizaram o protocolo composto de duas contrações isométricas balísticas máximas (CIBM) de dois segundos para os extensores do joelho e, três séries de contrações concêntricas (CC) para extensores e flexores do joelho. As CIBM foram realizadas a $60^{\circ}$ da articulação do joelho em relação ao solo. As séries de CC foram compostas da seguinte maneira: cinco repetições a $60^{\circ} . \mathrm{s}^{-1}$, 10 repetições a $180^{\circ} . \mathrm{s}^{-1}$ e 15 repetições a $300^{\circ} . \mathrm{s}^{-1}$. Durante o protocolo, foi utilizado um intervalo de três minutos entre séries (tabela 1).

Para a CIBM, os parâmetros analisados foram: impulso (IMP), pico de 
Tabela 1. Protocolo do teste realizado pelos atletas da Seleção Brasileira de Futebol de 5 no dinamômetro Isocinético.

\begin{tabular}{c|c|c|c|c}
\hline Velocidade & $\begin{array}{c}\text { Grupo } \\
\text { Muscular }\end{array}$ & $\begin{array}{c}\text { Tipo de } \\
\text { contração }\end{array}$ & $\mathbf{n}^{\text {* repetições }}$ & $\begin{array}{c}\text { Intervalo entre } \\
\text { as velocidades }\end{array}$ \\
\hline Isométrico & Extensores & Concêntrica & $2 \times 2 \mathrm{~s}$ & 120 s entre séries \\
\hline $60 \% \mathrm{~s}$ & $\begin{array}{c}\text { Flexores } \\
\text { Extensores }\end{array}$ & Concêntrica & 5 & $180 \mathrm{~s}$ \\
\hline $180 \% \mathrm{~s}$ & $\begin{array}{c}\text { Flexores } \\
\text { Extensores }\end{array}$ & Concêntrica & 10 & $180 \mathrm{~s}$ \\
\hline $300 \% \mathrm{~s}$ & $\begin{array}{c}\text { Flexores } \\
\text { Extensores }\end{array}$ & Concêntrica & 15 & $180 \mathrm{~s}$ \\
\hline
\end{tabular}

torque (PT) e pico de torque normalizado (PTN), que é a relação entre o PT e a massa corporal do atleta. Para as CC dos flexores e extensores do joelho, foram mensurados o PT, PTN, potência média (POM), quantidade total de trabalho (QTT), tempo para atingir o pico de torque (TPT) e o ângulo do pico de torque (APT). Ambos os exercícios foram realizados no membro dominante (MD) e membro não dominante (MND).

Calculou-se também a relação agonista/antagonista (RAA) entre os músculos flexores e extensores dominantes (MD) e não dominantes (MND) na velocidade angular de $60^{\circ} . \mathrm{s}^{-1}$, por meio da equação (RAA = PT dos flexores/PT dos extensores).

\section{Análise estatística}

Foi utilizada a estatística descritiva em média e desvio padrão para a caracterização dos sujeitos em relação à idade, variáveis antropométricas e de composição corporal. Para os resultados nas CIBM utilizou-se a média, o desvio padrão, os quartis 25 e 75, bem como os valores mínimos e máximos. Em seguida, foi realizado o teste de normalidade de Kolmogorov-Smirnov dos valores obtidos nas variáveis de composição corporal, nas CIBM e nas CC. Para comparação entre MD e MND nas diferentes velocidades analisadas, na CIBM e na RAA, utilizou-se o teste $t$ pareado e para comparação das variáveis antropométricas entre as posições utilizou-se o teste de ANOVA one-way.

Foi adotado o valor de $\mathrm{p} \leq 0,05$ para apontar as diferenças significativas. Todas as análises foram realizadas no programa GraphPad Prism $5^{\circledR}$ for Windows ${ }^{\circledR}$, versão 5.03 .

\section{RESULTADOS}

Na tabela 2, são descritas em média e desvio padrão as principais variáveis de caracterização antropométrica e de composição corporal dos atletas que compõe a Seleção Brasileira de Futebol de 5. Sob uma perspectiva geral, os pivôs e fixos, não apresentaram diferença estatística entre as variáveis de composição corporal. Porém, a EST e a MC dos alas quando comparadas com as dos fixos, apresentaram diferença entre os grupos. Tal variação pode estar relacionada aos aspectos morfológicos, exigidos pela modalidade em cada posição, no qual os alas são geralmente os jogadores com maior resistência aeróbia, para suportar maiores deslocamentos, enquanto que, os fixos são jogadores que tendem a apresentar níveis de força mais elevados.

Para avaliar o desempenho da musculatura flexora e extensora de joelho no membro dominante e não dominante, avaliou-se o PT, PTN,

Tabela 2. Caracterização em média e desvio padrão das variáveis antropométricas e de composição corporal dos atletas da Seleção Brasileira de Futebol de 5.

\begin{tabular}{c|c|c|c|c}
\hline \multirow{2}{*}{ Variáveis } & Pivô & Ala & Fixo & Geral \\
\cline { 2 - 5 } & $\mathbf{( n = 3 )}$ & $(\mathbf{n}=\mathbf{4})$ & $\mathbf{( n = 4 )}$ & $\mathbf{( n = 1 1 )}$ \\
\hline ID (anos) & $22,7 \pm 4,2$ & $25,3 \pm 4$ & $29,8 \pm 6,2$ & $25,9 \pm 3,6$ \\
\hline EST $(\mathrm{cm})$ & $172 \pm 0,04$ & $169 \pm 0,09^{*}$ & $179 \pm 0,06$ & $173 \pm 0,07$ \\
\hline MC $(\mathrm{Kg})$ & $76,4 \pm 13,7$ & $71,4 \pm 4,9^{*}$ & $79,3 \pm 4$ & $75,7 \pm 4$ \\
\hline IMC $\left(\mathrm{Kg} / \mathrm{m}^{2}\right)$ & $25,8 \pm 5$ & $25,2 \pm 2,5$ & $24,9 \pm 1,6$ & $25,3 \pm 2,1$ \\
\hline GC (\%) & $14,5 \pm 2,9$ & $12,7 \pm 4,2$ & $13,7 \pm 4,65$ & $13,6 \pm 0,9$ \\
\hline
\end{tabular}

ID - idade; EST - estatura; MC - massa corporal; IMC - Índice de massa corporal; GC - Gordura Corporal. * $\mathrm{p} \leq 0.05$ entre as posições de ala e fixo.
POM, QTT, TPT e APT nas velocidades angulares de $60^{\circ} . \mathrm{S}^{-1}, 180^{\circ} . \mathrm{S}^{-1} \mathrm{e}$ $300^{\circ} . \mathrm{S}^{-1}$ (tabela 3). No protocolo isométrico avaliou-se o PT, PTN e IMP referente à musculatura extensora do joelho (tabela 3). Todas as variáveis obtidas em ambos os protocolos foram mensuradas a fim de obter parâmetros para prevenir lesões em atletas de Futebol de 5. Na RAA entre a musculatura de MD e MND, encontrou-se valores próximos aos propostos pela literatura em esportes convencionais.

Nos movimentos concêntricos da musculatura flexora, foram observadas diferenças significativas $(p \leq 0,05)$ entre a variável PT nas velocidades de $60^{\circ} . \mathrm{s}^{-1}$ e $180^{\circ} . \mathrm{s}^{-1}$ entre o MD e o MND, sendo a diferença de $6,16 \%$ na velocidade de $60^{\circ} . \mathrm{s}^{-1}$ e de $4,91 \%$ na velocidade de $180^{\circ} . \mathrm{s}^{-1}$ O mesmo foi observado em relação ao PTN na velocidade de $60^{\circ} . \mathrm{s}^{-1}$ (5,75\%). Entre as variáveis PT, PTN e IMP entre MND e MD no exercício isométrico, não foi observada diferença estatística (tabela 4).

Para a RAA observou-se diferença significativa $(p \leq 0,05)$ entre MD e MND no exercício concêntrico com velocidade angular de $60^{\circ} . \mathrm{s}^{-1}$ (figura 1)

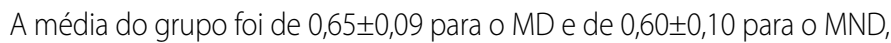
valores acima do limite inferior proposto para as velocidades mais lentas para se evitar lesões ${ }^{12}$.

Tabela 3. Valores em média e desvio padrão das variáveis neuromusculares obtidas no protocolo isocinético nas velocidades de 60,180 e $300^{\circ}$ s na pré-temporada de atletas da Seleção Brasileira de Futebol de 5.

\begin{tabular}{|c|c|c|c|c|c|c|c|}
\hline \multirow{2}{*}{ Variável } & \multirow{2}{*}{ Membro } & \multicolumn{3}{|c|}{ Flexores } & \multicolumn{3}{|c|}{ Extensores } \\
\hline & & $60^{\circ} . s-1$ & $180^{\circ} . s-1$ & $300^{\circ} . s-1$ & $60^{\circ} . s-1$ & $180^{\circ} . s-1$ & $300^{\circ} . s-1$ \\
\hline \multirow{2}{*}{$\begin{array}{c}\mathrm{PT} \\
(\mathrm{Nm})\end{array}$} & MND & $137 \pm 14^{*}$ & $116 \pm 8,5^{*}$ & $109 \pm 10,2$ & $232 \pm 36,6$ & $158 \pm 22,9$ & $127 \pm 18,4$ \\
\hline & $\mathrm{MD}$ & $146 \pm 15,4$ & $122 \pm 10,4$ & $113 \pm 11,2$ & $229 \pm 37,9$ & $154 \pm 19,7$ & $124 \pm 16,5$ \\
\hline \multirow{2}{*}{$\begin{array}{c}\text { PTN } \\
(\%)\end{array}$} & $M N D$ & $180 \pm 25,4^{*}$ & $152 \pm 17,1$ & $197 \pm 31$ & $303 \pm 47,2$ & $207 \pm 33,8^{*}$ & $143 \pm 18,4$ \\
\hline & $\mathrm{MD}$ & $191 \pm 22,5$ & $155 \pm 16,2$ & $165 \pm 24$ & $292 \pm 52,7$ & $197 \pm 30,9$ & $159 \pm 27$ \\
\hline \multirow{2}{*}{$\begin{array}{l}\text { POM } \\
\text { (W) }\end{array}$} & MND & $104 \pm 29,2$ & $260 \pm 64,6^{*}$ & $242 \pm 27,5$ & $154 \pm 46,4$ & $215 \pm 29,6$ & $256 \pm 48,6$ \\
\hline & $M D$ & $101 \pm 28,1$ & $215 \pm 29,6$ & $249 \pm 37,6$ & $144 \pm 34,1$ & $256 \pm 48,6$ & $277 \pm 48$ \\
\hline \multirow{2}{*}{$\begin{array}{l}\mathrm{QTT} \\
(J)\end{array}$} & MND & $506 \pm 172,9$ & $1094 \pm 117,5$ & $1468 \pm 111,8$ & $697 \pm 219$ & $1236 \pm 208,2$ & $1519 \pm 211,2$ \\
\hline & $\mathrm{MD}$ & $547 \pm 171,9$ & $1129 \pm 119,6$ & $1504 \pm 178,7$ & $703 \pm 200,5$ & $1225 \pm 189,4$ & $1490 \pm 181,7$ \\
\hline \multirow{2}{*}{ TPT (ms) } & $M N D$ & $420 \pm 127,9$ & $231 \pm 100,3$ & $224 \pm 64,3$ & $376 \pm 50$ & $154 \pm 25,9$ & $110 \pm 12,4$ \\
\hline & $\mathrm{MD}$ & $388 \pm 96,4$ & $243 \pm 98,8$ & $212 \pm 75,1$ & $343 \pm 61,2$ & $147 \pm 31,0$ & $109 \pm 12,9$ \\
\hline \multirow{2}{*}{ APT (०) } & MND & $54 \pm 7,4$ & $51 \pm 14,6$ & $64 \pm 16,6$ & $64 \pm 3,1$ & $60 \pm 4,1$ & $62 \pm 2,4$ \\
\hline & $\mathrm{MD}$ & $51 \pm 9,2$ & $54 \pm 13,4$ & $61 \pm 17,8$ & $65 \pm 2,6$ & $62 \pm 4,9$ & $62 \pm 2,4$ \\
\hline
\end{tabular}

PT - pico de torque; PTN - pico de torque normalizado (PT/MC): POM - potência média; QTT - quantidade tota de trabalho; TPT - tempo para atingir pico de torque; APT - ângulo do pico de torque.*diferença estatística $(p \leq 0,05)$ entre o MND e o MD.

Tabela 4. Valores em média e desvio padrão das variáveis neuromusculares obtidas no protocolo isométrico na pré-temporada de atletas da Seleção Brasileira de Futebol de 5 .

\begin{tabular}{c|c|c|c|c}
\hline Membro & \multicolumn{2}{|c|}{ MND } & \multicolumn{2}{c}{ MD } \\
\hline Variavéis & PT (Nm) & PTN (\%) & PT (Nm) & PTN (\%) \\
\hline m $\pm d p$ & $248 \pm 47,8$ & $324,6 \pm 63,3$ & $249,1 \pm 45,3$ & $325 \pm 53,8$ \\
\hline mín & 170,3 & 223,2 & 178 & 225,3 \\
\hline Q25 & 223,9 & 278,7 & 233,2 & 296,1 \\
\hline Q75 & 270,1 & 371,4 & 260,3 & 356,1 \\
\hline máx & 332,3 & 438,2 & 329,4 & 425,3 \\
\hline
\end{tabular}

PT - Pico de Torque: PTN - Pico de Torque Normalizado (PT/MC) IMP - Impulso; MND - Membro não dominante; $\mathrm{MD}$ - Membro Dominante. *diferença entre os MND e MD $(p \leq 0,05)$.

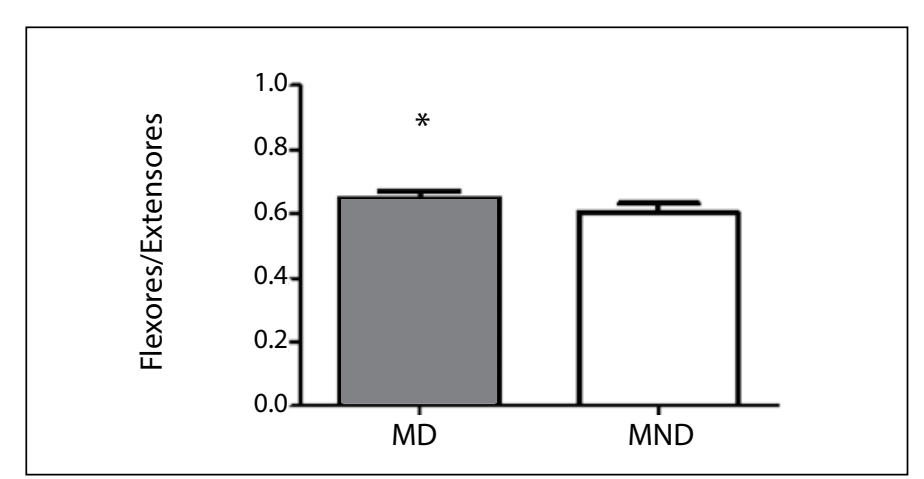

Figura 1. Razão convencional dos membros dominantes (MD) e não dominantes (MND) de atletas da seleção brasileira de futebol de 5 . 


\section{DISCUSSÃO}

No presente estudo, buscamos avaliar o desempenho neuromuscular da musculatura extensora e flexora do joelho e mensurar a relação entre musculatura agonistas e antagonista dos membros inferiores, em atletas de elite de Futebol de 5. Apesar de indivíduos com deficiência visual apresentarem um equilíbrio muscular inferior aos sujeitos sem deficiência, sendo mais suscetíveis a lesão muscular ${ }^{1,2}$, não observamos tais alterações neste grupo de atletas. Devido a não existência de publicações de valores normativos para os parâmetros neuromusculares em atletas com deficiência visual praticantes de futebol de 5, os valores do nosso estudo foram comparados com os valores normativos estabelecidos para o futebol convencional. Dessa forma, observamos valores similares aos que são aceitáveis, no futebol convencional, na variável de RAA e na diferença entre o MD e MND 5,13,14. Além disso, não observamos diferença contralateral maior do que o limite máximo proposto para indivíduos sem deficiência no $\mathrm{PT}$ a $60^{\circ} \mathrm{s}^{-1}$ pelos MD e $M N D^{15}$. Visto que a propriocepção em indivíduos com deficiência visual é comprometida em função da ausência dos ajustes cálculo-óptico-motor e da menor coordenação intra e intermuscular, o índice de lesão nestes indivíduos pode ser aumentado, além da produção de força ser afetada ${ }^{16}$. Neste sentido, é possível que os valores de RAA, PT, PTN observados no presente estudo, estejam relacionados ao treinamento sistematizado ao quais estes atletas foram submetidos. No caso dos atletas incluídos neste estudo, o treinamento utilizado neste grupo é específico para a modalidade, com o objetivo de reduzir o desequilíbrio muscular nestes atletas, e dessa forma, diminuir a susceptibilidade a lesão por sobrecarga e aumento do desempenho em quadra.

Cabe ressaltar que apesar da observação de valores aceitáveis para o déficit bilateral entre membros ${ }^{15}$ e RAA ${ }^{17}$, o PT, PTN, POM e RAA foram superiores no MD quando comparados ao MND. Tais parâmetros são associados à velocidade de aceleração que é o desempenho na fase inicial da corrida, conhecida também como "arrancada", sendo determinante para a intensidade de jogo ${ }^{13}$. Assim, apesar dos valores de equilíbrio muscular estarem dentro do recomendado, nota-se um desequilíbrio entre MD e MND, que pode comprometer em parte algumas exigências específicas da modalidade que necessitam da utilização de ambos os membros.

Uma limitação do estudo é que em virtude da escassez de estudos que caracterizem RAA, PT, PTN e POM em atletas com deficiência visual, não foi possível comparar os presentes resultados com valores de outros estudos, com o objetivo de investigar possíveis determinantes de variações no desempenho e desequilibrio muscular. Porém, o fato do estudo ser pioneiro na avaliação dos níveis de torque máximo, da diferença bilateral na produção de força e a razão convencional das musculaturas flexoras e extensoras do joelho em diferentes velocidades de execução em atletas praticantes de futebol de 5, nossos achados indicam que os atletas com deficiência visual, apresentaram níveis aceitáveis da razão convencional, de acordo com o proposto para o futebol convencional ${ }^{5,13,14}$ apesar da diferença observada na variável de RAA entre os MD e MND.

\section{CONCLUSÃO}

Os resultados obtidos neste estudo fornecem valores do desempenho muscular isocinético da musculatura extensora e flexora do joelho, em diferentes velocidades de execução dos atletas de elite de futebol de 5. Além da caracterização do perfil muscular dos atletas, mensurou-se a relação convencional entre a musculatura agonistas e antagonista dos membros MD e MND. Nessa comparação foram observadas diferença significativa entre MD e MND, porém, níveis aceitáveis em ambas as pernas de acordo com os parâmetros estabelecidos para o futebol convencional. Nesse sentido, espera-se que os resultados encontrados possam contribuir para os processos de prevenção, treinamento e reabilitação de atleta de futebol de 5, e além disso, que esses dados possam servir como parâmetros para futuros estudos que objetivem relacionar as variáveis aqui utilizadas. De forma generalizada, acreditamos que o próximo desafio para a respectiva modalidade, é o monitoramento das variáveis neuromusculares durante todo o período de preparação física, associada ao conteúdo específico de treinamento, como também, estabelecer valores normativos por posição.

\section{AGRADECIMENTOS}

Ao Conselho Nacional de Desenvolvimento Científico e Tecnológico - CNPq e a Confederação Brasileira de Desportos para Deficientes Visuais - CBDV.

Todos os autores declararam não haver qualquer potencial conflito de interesses referente a este artigo.

\section{REFERÊNCIAS}

1. Silva MPM, Duarte E, Costa e Silva AA, Silva HGPV, Vital R. Aspectos das lesões esportivas em atletas com deficiência visual. Rev Bras Med Esporte. 2011;17(5): 319-23.

2. Magno e Silva MP, Morato MP, Bilzon JL, Duarte E. Sports injuries in Brazilian blind footballers. Int J Sports Med. 2013;34(3):239-43

3. Terreri ASA, Greve JMD, Amatuzzi MM. Avaliação isocinética no joelho do atleta. Rev Bras Med Esporte. 2001;7(5):170-4.

4. Silva Neto M, Simões R, Grangeiro Neto JA, Cardone CP. Isokinetic Assessment of Muscle Strength in Female Professional Soccer Athletes. Rev Bras Med Esporte. 2010;16(1): 33-5.

5. Zabaka FF, Valente HG, Pacheco AM. Isokinetic evaluation of knee extensor and flexor muscles in professional soccer players. Rev Bras Med Esporte. 2011;17(3):189-192.

6. Carvalho P, Cabri J. Avaliação isocinética da força dos músculos da coxa dos futebolistas. Rev Port Fisioter Desporto. 2007;1(2):4-12

7. Ferreira AP, Gomes SA, Ferreira CES, Arruda M, França NM. Avaliação do desempenho isocinético da musculatura extensora e flexora do joelho de atletas de futsal em membro dominante e não dominante. Rev Bras Ciênc Esporte. 2010;32(1):229-43.

8. Weber FS, Silva BGC, Cadore EL, Pinto SS, Pinto RS. Isokinetic assessment in professional soccer players and performance comparison according to their different positions in the field. Rev Bras Med Esporte. 2010;16(4):264-8.

9. Campos LFC, Costa e Silva AA, Santos LGT, Costa LT, Montagner PC, Borin JP, et al. Effects of training in physical fitness and body composition of the brazilian 5-a-side football team. Rev And Med Deporte. 2013;6(3):91-5

10. Issurin VB. New horizons for the methodology and Physiology of training periodization. Sports Med. 2010;40(3):189-206

11. Jackson AS, Pollock ML. Generalized equations for predicting body density of men. Br J Nutr. 1978;40(3):497-504

12. Siri WE. Body composition from fluid space and density. In: Brozek J, Hanschel A, editors. Techniques for measuring body composition. Washigton, DC: National Academy of Science;1961. p. 223.

13. Fonseca ST, Ocarino JM, Silva PLP, Bricio RS, Costa CA, Wanner LL. Caracterização da performance muscular em atletas profissionais de futebol. Rev Bras Med Esporte. 2007;13(3):143-7.

14. Kannus P. Isokinetic evaluation of muscular performance: implications for muscle testing and rehabilitation. Int J Sports Med. 1994;15(Suppl 1):11-8.

15. Reid DC. Sports injury, assessment and rehabilitation. New York, NY: Churchill Livingstone; 1992.

16. Bosco CA. A força muscular: aspectos fisiológicos e aplicações práticas. São Paulo, SP: Phorte; 2007.

17. Andrews JR, Harrelson GL, Wilk KE. Reabilitação física das lesões desportivas. 3a. ed. Rio de Janeiro: Guanabara; 2005 